\title{
Equalization: Analysis of MIMO Systems in Frequency Selective Channel
}

\author{
Amit Grover ${ }^{1 *}$ \\ Assistant Professor, Department of ECE \\ Shaheed Bhagat Singh State Technical Campus, \\ Moga Road (NH-95), Ferozepur -152004, India.
}

\begin{abstract}
Due to the increased demand of wireless communication systems because of the features of the system which provides a wide coverage, high throughput and reliable services, the MIMO systems communication has come into existence. Features provided by these systems ensure the improved system coverage and increased data transmission rate by considering multiple numbers of transmitter and receiver antennas. In this paper, we are considering the equalization; a filtering approach that minimizes the error between actual output and desired output by continuous updating its filter coefficients for Rayleigh Frequency selective fading channel. We concluded that MMSE and ZF give the worst performance in Rayleigh frequency selective channel as compare to Rayleigh Flat fading Channel [35] due to a constant BER for large SNR's. We have also observed that the successive interference methods provide better performance as compare to others, but their complexity is high. ML provides the better performance in comparison to others and BER doesn't remain constant for a large SNR in this case. Simulation results shows that ML equalizer with BPSK gives better performance as compare to QPSK. Finally we concluded that Sphere decoder provides the best performance.
\end{abstract}

Keywords-Quadrature Amplitude Modulation (QAM); Quadrature Phase Shift Key (QPSK); Binary Phase Shift Key (BPSK); Minimum mean-squared error (MMSE); Maximum likelihood $(M L) ; \quad B i t$ error rate (BER); Inter-symbol interference (ISI); Successive-interference-cancellation (SIC); Sphere Decoder (SD); Zero Forcing $(\mathrm{ZF})$.

\section{INTRODUCTION}

Because of the features of MIMO systems, it became an important part of modern wireless communication [5]. Communication in wireless channels is impaired predominantly by multipath fading. Multipath is the arrival of the transmitted signal at the receiver through differing angles and/or differing time delays and/or differing frequency [4]. MIMO offers significant increases in data throughput and link range without additional bandwidth or transmit power. It achieves this by higher spectral efficiency and link reliability and or diversity. The information bits to be transmitted are encoded and interleaved [9]. The Symbol mapper mapped the interleaved codeword's to data symbols and then these data symbols are applied as input to the Space Time-encoder which are again mapped to transmit antennas by space-time pre-coding block and are received at the antenna array by passing through the channel. The receiver performs the reverse operation to that of transmitter, followed by space time decoding [7].

\section{MIMO SYSTEM MODEL}

By considering a MIMO system with a transmit array of $M_{T}$ antennas and a receive array of $\mathrm{M}_{\mathrm{R}}$ antennas [11], the MIMO channel [35] at a given time instant may be represented as a $M_{R} \times M_{T}$ matrix

$$
\begin{array}{r}
\mathrm{H}=\left[\begin{array}{cccc}
\mathrm{H}_{1,1} & \mathrm{H}_{1,2} & \cdots & \mathrm{H}_{1, \mathrm{M}_{\mathrm{T}}} \\
\mathrm{H}_{2,1} & \mathrm{H}_{2,2} & \cdots & \mathrm{H}_{2, \mathrm{M}_{\mathrm{T}}} \\
\vdots & \vdots & \ddots & \vdots \\
\mathrm{H}_{\mathrm{M}_{\mathrm{R}}, 1} & \mathrm{H}_{\mathrm{M}_{\mathrm{R}}, 2} & \cdots & \mathrm{H}_{\mathrm{M}_{\mathrm{R}}, \mathrm{M}_{\mathrm{T}}}
\end{array}\right] \\
\text { III. } \\
\text { MIMO CHANNEL }
\end{array}
$$

By assuming the above channel model the product of the bandwidth and the delay spread is very small and it results in channel realizations which varies with the time and is frequency dependent that is $\mathrm{H}$ (f) [35].

\section{EQUALIZATION TECHNIQUES}

\section{A. Zero forcing}

Zero forcing is a linear equalization method that does not consider the effects of noise. In fact, the noise may be enhanced in the process of eliminating the interference. A zero-forcing equalizer uses an inverse filter to compensate for the channel response function [4].By assuming $\mathrm{M}_{T}=\mathrm{M}_{R}$ and $\mathrm{H}$ is a full rank square matrix, we can calculate the covariance matrix [35] of the effected noise as:

$$
E\left[\left(n \mathrm{H}^{-1}\right)^{H} \cdot n \mathrm{H}^{-1}\right]=\left(H^{-1}\right)^{H} \cdot E\left[n^{H} \cdot n\right] \cdot H^{-1}=n\left(H \cdot H^{H}\right)^{-1}
$$

\section{B. Minimum mean square error (MMSE)}

The MMSE detector is the optimal detection that seeks to balance between cancelation of the interference and reduction of noise enhancement. We assume that the number of receive antennas is less than the number of transmit antennas $\mathrm{M} \geq \mathrm{N}$ MMSE at a high SNR [35] is given by

$$
\mathrm{W}_{\mathrm{MMSE}}=\left(\mathrm{H}^{*} \mathrm{H}+\frac{1}{\mathrm{SNR}} \mathrm{I}\right)^{-1} \mathrm{H}^{*} \approx\left(\mathrm{H}^{\mathrm{H}} \mathrm{H}\right)^{-1} \mathrm{H}^{\mathrm{H}}
$$

\section{Successive Interference Cancelation.}

The SIC schemes also reduces the noise amplification by the nulling vector. Therefore after the first cancelation the nulling vector for the second stream need only $M_{r}-1$ dimensions. The performance can also be enhanced by optimal ordering the SIC process, in which a nulling vector has been 
chosen that has the smallest norm to detect the corresponding data stream, but the system becomes more complicated.

\section{Maximum Likelihood (ML)}

Maximum likelihood detection [35] calculates the Euclidean distance between received signal vector and the product of all possible transmitted signal vectors with the given channel $\mathrm{H}$, and finds the one with minimum distance.

The ML method achieves the optimal performance as the maximum a posterior detection when all the transmitted vectors are likely. However, its complexity increases exponentially as modulation order and/or the number of transmit antennas increases [6]. The ML receiver performs optimum vector decoding and is optimal in the sense of minimizing the error probability.

\section{E. Sphere Decoder (SD)}

By using sphere decoding, a limited number of codeword's are considered that are within a sphere centered at the received signal vector. By using this technique we find the ML solution vector that considers only a small set of vectors within a given sphere rather than all possible transmitted signal vectors [2]. By Considering the sphere with radius of $\mathrm{R}_{\mathrm{SD}}$ as shown in equation (4)

$$
(\overline{\mathrm{x}}-\hat{\mathrm{x}})^{\mathrm{T}} \overline{\mathrm{H}}^{\mathrm{T}} \overline{\mathrm{H}}(\overline{\mathrm{x}}-\hat{\mathrm{x}}) \leq \mathrm{R}_{S \mathrm{D}}^{2}
$$

$\mathrm{SD}$ method considers only the vectors inside a sphere defined by Equation (4). By considering the example of sphere which includes four candidate vectors, one of which is the ML solution vector. No vector outside the sphere can be the ML solution vector because their ML metric values are bigger than the ones inside the sphere [16]. If we were fortunate to choose the closest one among the four candidate vectors, we can reduce the radius in Equation (4) so that we may have a sphere within which a single vector remains. In other words, the ML solution vector is now constrained in this sphere with a reduced radius [35]. And we can express the Equation (4) as

$$
\begin{aligned}
& =\left|r_{44}\left(\bar{x}_{4}-\hat{\bar{x}}_{4}\right)\right|^{2}+\left|r_{33}\left(\bar{x}_{3}-\hat{\bar{x}}_{3}\right)\right|^{2}+\left|r_{34}\left(\bar{x}_{4}-\hat{\bar{x}}_{4}\right)\right|^{2} \\
& +\left|\mathrm{r}_{22}\left(\overline{\mathrm{x}}_{2}-\hat{\overline{\mathrm{x}}}_{2}\right)\right|^{2}+\left|\mathrm{r}_{23}\left(\overline{\mathrm{x}}_{3}-\hat{\overline{\mathrm{x}}}_{3}\right)\right|^{2} \\
& +\left|\mathrm{r}_{24}\left(\overline{\mathrm{x}}_{4}-\hat{\mathrm{x}}_{4}\right)\right|^{2}+\left|\mathrm{r}_{11}\left(\overline{\mathrm{x}}_{1}-\hat{\mathrm{x}}_{1}\right)\right|^{2} \\
& +\left|\mathrm{r}_{12}\left(\overline{\mathrm{x}}_{2}-\hat{\mathrm{x}}_{2}\right)\right|^{2}+\left|\mathrm{r}_{13}\left(\overline{\mathrm{x}}_{3}-\hat{\mathrm{x}}_{3}\right)\right|^{2} \\
& +\left|\mathrm{r}_{14}\left(\overline{\mathrm{x}}_{4}-\hat{\mathrm{x}}_{4}\right)\right|^{2} \leq \mathrm{R}_{\mathrm{SD}}^{2}
\end{aligned}
$$

Using the Sphere in Equation (5), we consider a candidate value for $\hat{\mathrm{x}}_{4}$ in its own single dimension, which is arbitrarily chosen from the points in the sphere and this point, must be chosen in the following range:

$$
\hat{\mathrm{x}}_{4}-\frac{\mathrm{R}_{\mathrm{SD}}}{\mathrm{r}_{44}} \leq \overline{\mathrm{x}}_{4} \leq \hat{\mathrm{x}}_{4}+\frac{\mathrm{R}_{\mathrm{SD}}}{\mathrm{r}_{44}}
$$

If there exists no candidate point satisfying the inequalities, the radius needs to be increased. We assume that a candidate value was successfully chosen. Then we proceed to next step. We consider a candidate value for $\bar{x}_{3}$ now. If a candidate value for $\overline{\mathrm{x}}_{3}$ does not exist, we go back to Step 1 and choose other candidate value of $\tilde{\bar{x}}_{4}$ and search for $\overline{\mathrm{x}}_{3}$. In case that no candidate value $\overline{\mathrm{x}}_{3}$ exists with all possible values $\tilde{\overline{\mathrm{x}}}_{4}$, we increase the radius of sphere $\mathrm{R}_{\mathrm{SD}}$, and repeat the step 1 . Similarly a candidate value for $\overline{\mathrm{x}}_{1}$ is chosen. After finding all candidate values, radius can be calculated by Equation (5).

Using this new radius Step 1 is repeated. If $\left[\begin{array}{llll}\tilde{\overline{\mathrm{x}}}_{1} & \tilde{\overline{\mathrm{X}}}_{2} & \tilde{\overline{\mathrm{x}}}_{3} & \tilde{\overline{\mathrm{x}}}_{4}\end{array}\right]$ turns out to be a single point inside a sphere with that radius, it is declared as the ML solution vector [35] and searching procedure stops.

\section{Simulation And Results}

Using MATLAB, the different simulation results are shown in the different graphs, which provide the comparison of the BER for different modulation techniques using different equalizers like MMSE, ZF, ZF-SIC, MMSE-SIC and ML with Rayleigh frequency selective fading channel.

"Fig.4"and"Fig.5"shows the comparison of BER for different modulations with MMSE and ZF equalizers.

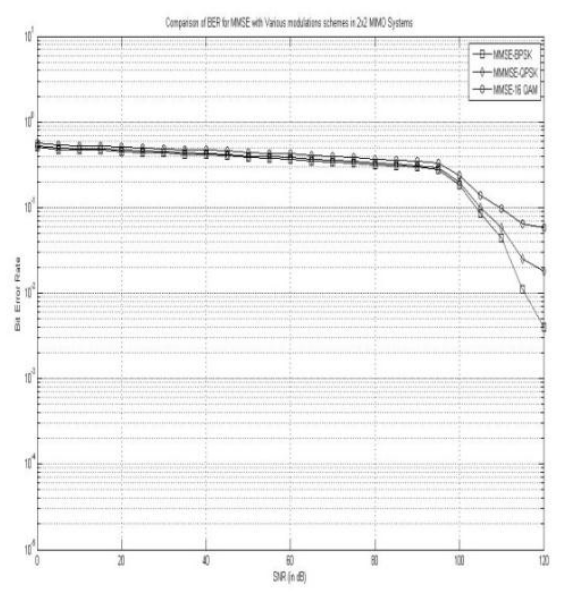

Figure 4.

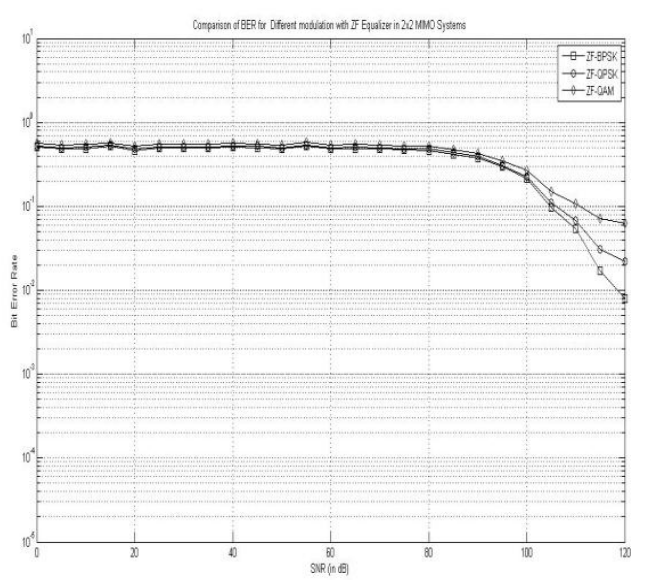

Figure 5.

The MMSE and ZF equalizer doesn't work well in Frequency selective fading channel. As it is clear from the "Fig.4" and "Fig.5" that BER remains constant for a large SNR and a deviation in BER is there at large SNR, and the performance of MMSE is little bit better in comparison to ZF. So the simulation of MMSE and ZF equalizer with BPSK by 
using MIMO 2 x2 Frequency selective fading channel, gives the better performance as compared to QPSK and 16QAM in both the cases.

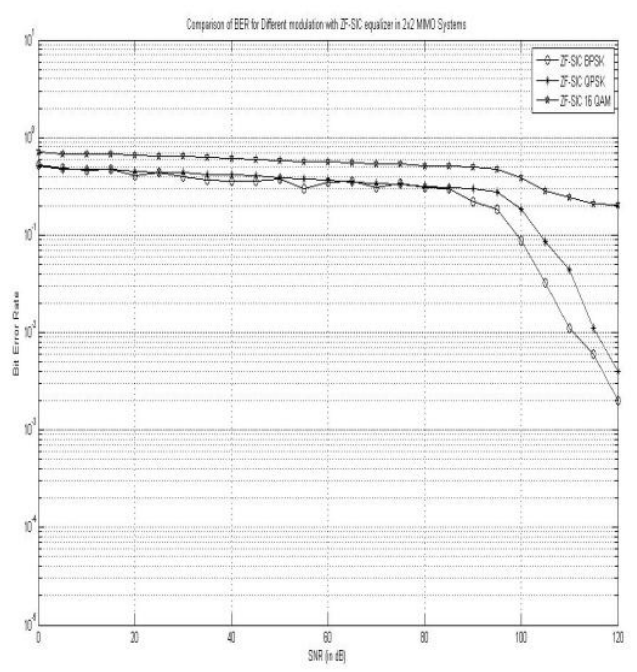

Figure 6.

"Fig.6"shows the comparison of BER for different modulations with ZF-SIC equalizer.

The ZF-SIC and MMSE-SIC equalizer doesn't work well in Frequency selective fading channel. As it is clear from the "Fig.6" and "Fig.7" BER remains constant for a large SNR and a deviation in BER is there at large SNR.

The performance of ZF-SIC is little bit better in comparison to ZF. So the simulation of ZF-SIC and MMSE-SIC equalizer with BPSK by using MIMO 2 x2 Frequency selective fading channel, gives the better performance as compared to QPSK and 16 QAM.

"Fig.7"shows the comparison of BER for different modulations with MMSE-SIC equalizer.

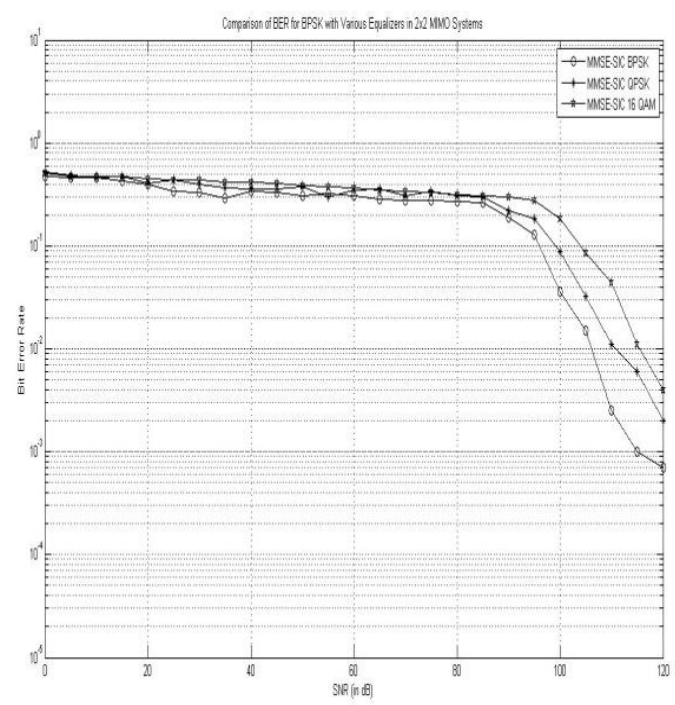

Figure 7.

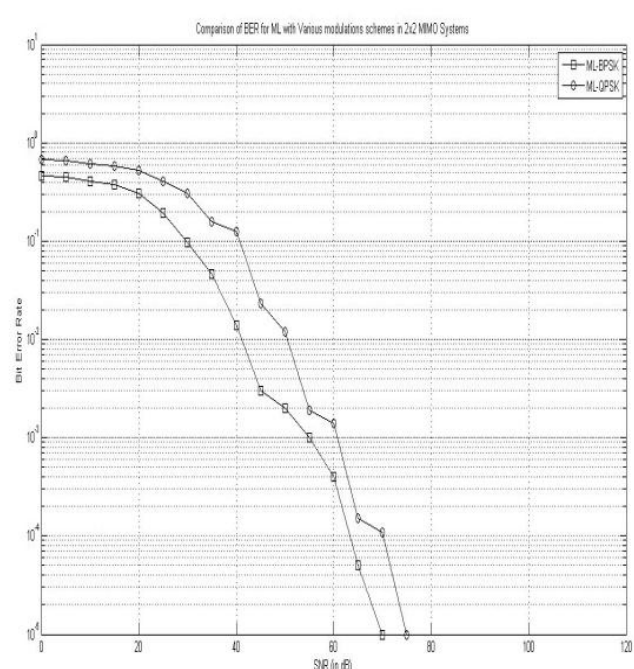

Figure 8.

"Fig.8"shows the comparison of BER for different modulations with ML equalizer.

The ML equalizer works well in Frequency selective fading channel in comparison to previously discussed equalizers. As it is clear from "Fig.8" BER doesn't remain constant for a large SNR as in another case. We can conclude that ML equalizer gives us good result in frequency fading. When we simulated ML equalizer with BPSK and QPSK by using MIMO $2 \times 2$ Frequency selective fading channel, then as expected the performance of BPSK is better than QPSK.

"Fig.9"shows the comparison of all the equalizers with their best modulation scheme, and the simulation result for transmitting 2 bits/sec over two transmit and two receive antennas using BPSK. The results are decoded using the ZF, MMSE, ZF-SIC, MMSE-SIC, ML and Sphere decoder (SD) technique. The successive interference methods outperform the $\mathrm{ZF}$ and MMSE however their complexity is higher due to iterative nature of the algorithms.

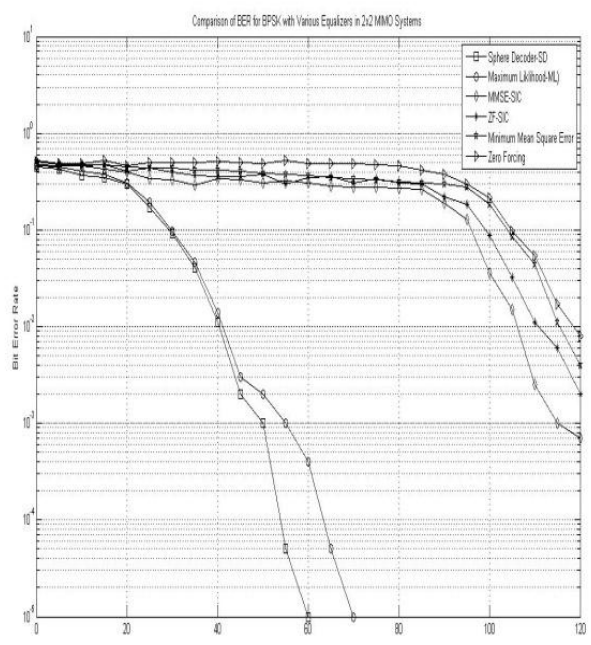

Figure 9. 
"Fig.9"shows the comparison of BER for BPSK with different equalizers.

\section{CONCLUSIONS}

When equalization is done through frequency selective fading channel, then the performance of ZF, MMSE, ZF-SIC and MMSE-SIC are very poor. ML provides the better performance in comparison to other equalizers. Sphere decoder provides the best performance and the highest decoding complexity as compare to ML. BER performance in order to highest to lowest is as: $\mathrm{SD}>\mathrm{ML}>\mathrm{MMSE}-\mathrm{SIC}>\mathrm{ZF}-\mathrm{SIC}>$ MMSE > ZF. We finally concluded that SD is best suited method to remove ISI in Frequency selective fading channel in MIMO systems.

\section{REFERENCES}

[1] [1] H. El Gamal and A.R. Hammons, "The layered space-time architecture: a new perspective", IEEE Trans. Inform. Theory, vol. 47, pp. 2321-2334, Sept. 2001.

[2] Simon, M. K. and Alouini, M. Digital Communication over Fading Channels. John Wiley \& Sons, 2004.

[3] G.J. Foschini and M.J. Gans, "On limits of wireless communications in a fading environment when using multiple antennas", Wireless Personal Communications, vol. 6, pp. 311-335, 1998.

[4] G.J. Foschini, "Layered space-time architecture for wireless communications in a fading environment when using multiple antennas", Bell Labs. Tech. J., vol. 6, no. 2, pp. 41-59, 1996.

[5] M. Janakiraman ("Space-time codes and MIMO systems", Artech House, 2004.

[6] J .C. Liberti and T. S. Rappaport "Smart Antennas for Wireless Communications, Prentice H all PTR, 1999.

[7] Wang and G. B. Giannakis, "A simple and general parameterization quantifying performance in fading channels," IEEE Trans. Communication., vol. 51, no. 8, pp. 1389-1398, 2003.

[8] J. G. Proakis, Digital Communications, McGraw-Hill series in electrical and computer engineering, 1995.

[9] Ezio Biglieri, Robert Calderbank, Anthony Constantinides, Andrea Goldsmith, Arogyaswami Paulraj, H.Vincent Poor "MIMO Wireless Communications", Cambridge University Press, (2007).

[10] G. Arslan, B. L. Evans, and S. Kiaei , "Equalization for Discrete Multitone Receivers To Maximize Channel Capacity", IEEE Transactions on Signal Processing, submitted March 30, 2000.

[11] D. Shiu P. J. Smith D. Gesbert, M. Shafi and A. Nayguib, "From theory to practice: An overview of MIMO space-time coded wireless systems," IEEE J. Select. Areas Commun. vol., 21, no. 3, pp. 281-302, 2003.

[12] R. U. Nabar A. J. Paulraj, D. A. Gore and H. Bolcskei, "An overview of MIMO communications - a key to gigabit wireless," Proceedings of the IEEE, vol. 92, no. 2, pp. 198-218, 2004.

[13] G.Ginis and J.M.Cioffi, "On the relationship between V-BLAST and GDFE," IEEE Communications letters, vol. 5, pp. 364-366, 2001.

[14] L. Zheng and D. Tse, "Diversity and multiplexing: A fundamental tradeoff in multiple-antenna channels," IEEE Transactions on Information Theory, vol. 49, pp. 1073-1096, May 2003.

[15] G. D. Golden , G. J. Foschini, R. A. Valenzuela and P. W. Wolniansky, “ Detection algorithm and initial laboratory results using v-blast spacetime communication architecture,"IEEE Electronic Letters, Vol.35, No.1, pp.14 16, January 1999.

[16] J. Zhang, T. Bhatt, G. Mandyam "Efficient linear equalization for high data rate downlink CDMA signaling", 37th IEEE Asilomar Conference on Signals, Systems and Computers, 2003.

[17] V. K. Garg and J .E. Wilkes, "Wireless and Personal Communications Systems’ Prentice Hall, 1996.
[18] Gesbert "MIMO space-time coded wireless systems,"presentation available at http://www.tele.ntnu.no/projects/beats/course.htm. Sept. 2003.

[19] D. Shiu and J. M. Kahn "Layered space-time codes for wireless communications using multiple transmit antennas," Vancouver, Canada, 1999.

[20] G. Foschini, G. Golden, R. Valenzuela and P. Wolniansky "Simplified processing for high spectral efficiency wireless communication employing multi-element arrays", IEEE Journal on Selected Areas in Communications, vol. 17, pp. 1841-1852, 2000.

[21] D. Wubben, R. Bohnke, J. Rinas, V. Kuhn and K .D. Kammeyer "Efficient algorithm for decoding layered s pace-time codes", Electronics Letters, vol. 37, pp. 1348-1350, 2001.

[22] K.Lo, S.Marinkovic, Z C hen and B.Vucetic "BER performance comparison of layered space time codes", New York, USA ICC 2002.

[23] Choo,Y,S, ,Kim,J.,Yang,W.Y., and Kang C.G "Mimo-OFDM Wireless communication with matlab", IEEE PRESS, John Wiley and sons (Asia) Pte Ltd.

[24] C. E. Proakis, "Digital Communications," McGraw-Hill International Editions, New York, 4th edition, 2000.

[25] H. Jafarkhani "Space-time coding: Theory \& Practice", Cambridge University Press, 2005.

[26] I.E. Telatar "Capacity of multi-antenna Gaussian channels, "European Transactions on Telecommunications, vol. 10, no.6, pp.585-595, 1999.

[27] S.Loyka and F. Gagon "Performance analysis of the V-BLAST algorithm: an analytical approach, "IEEE Transactions on Wireless Communications. Vol. 3, pp. 1326-1337, July 2004.

[28] K.I.Pedersen, J.B.Anderson, J.P.Kermoal and P.E.Mogensen "A stochastic multiple-input multiple-output radio channel model for evaluation of space-time coding algorithms," in Proc. VTC 200 Fall, Boston, vol. 2, pp.893-897, Sep. 2000.

[29] M.Varanasi and T.Guess "Optimum decision feedback multiuser equalization with successive decoding achieves the total capacity of the Gaussian multiple-access channel," Conference Record of the ThirtyFirst Asilomar Conference on signals, Systems and computers, vol. 2, pp. 1405-1409, Nov-2-5 1997.

[30] A.M.Tulino and S.Verdu "Random Matrix Theory and Wireless Communications, Hanover MA 02339, USA: now publishers Inc., 2004.

[31] E.Biglieri, J.Proakies and S.Shamai 'Fading Channel Information Theoretic and Communication Aspects", IEEE Trans. On information Theory, vol. 44, pp.2619-2692, Oct. 1998.

[32] X.Li, H.Huang, G.J.Foschini, and R.A.Valenzu "Effects of Iterative Detection and Decoding on the Performance of BLAST", IEEE Global Telecommunications Conference, vol.2, pp.1061-10066, Nov 2000.

[33] David Tse and Pramod Viswanath," Fundamentals of Wireless Communication Cambridge University Press, 2005.

[34] John G. Proakis and Masoud Salehi "Contemporary Communication Systems using Matlab," Brooks/Cole, 2000.

[35] Rohit Gupta, Amit Grover," BER Performance Analysis of MIMO Systems Using Equalization Techniques",Innovative System Design and Engineering, vol.3, no.10, pp. 11-25, 2012.

\section{AUTHORS PROFILE}

Amit Grover(M'06-SM'09- PI'11\&12 ) The author became a Member (M) of Association ISTE in 2006, a Senior Member (SM) of society SELCOME in September 2009, and a Project-In charge (PI) in august 2011 and in September 2012. The author place of birth is Ferozepur, Punjab, India on 27th, September 1980.

The author received M. Tech degree in Electronics and

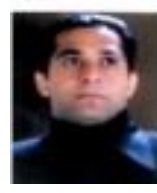

Engineering from Punjab Technical University, Kapurthla, Punjab, India in 2008 and received B. Tech degree in Electronics and Communication Engineering from Punjab Technical University, Kapurthala, Punjab, India in 2001. Currently, he is working as an Assistant Professor in Shaheed Bhagat Singh State Technical Campus, Ferozepur, Punjab. His area of interest includes signal processing, MIMO systems, wireless mobile communication, high speed digital communications and $4 \mathrm{G}$ wireless communications. 